Cahiers de recherches médiévales

\title{
Savoirs et pratiques diététiques au Moyen Âge
}

\section{Marilyn Nicoud}

\section{(2) OpenEdition \\ Journals}

Édition électronique

URL : https://journals.openedition.org/crm/864

DOI : $10.4000 / \mathrm{crm} .864$

ISSN : 1955-2424

Éditeur

Honoré Champion

\section{Édition imprimée}

Date de publication : 30 mars 2006

Pagination : 239-247

ISSN : $1272-9752$

\section{Référence électronique}

Marilyn Nicoud, "Savoirs et pratiques diététiques au Moyen Âge », Cahiers de recherches médiévales [En ligne], 13 spécial | 2006, mis en ligne le 03 avril 2009, consulté le 15 décembre 2022. URL : http:// journals.openedition.org/crm/864 ; DOI : https://doi.org/10.4000/crm.864 


\section{酷M}

\section{Savoirs et pratiques diététiques au Moyen Âge}

L'appartenance de la diététique au domaine de compétence du praticien médiéval est affirmée par nombre d'ouvrages utilisés pour l'enseignement de la médecine, à commencer par le Canon d'Avicenne, véritable somme du savoir médical, traduit en latin à Tolède au XII ${ }^{\mathrm{e}}$ siècle par Gérard de Crémone. Avicenne y rappelle que la médecine est divisée en deux branches : l'une est dite théorique (car elle a pour finalité la connaissance des causes de la santé et de la maladie); l'autre pratique - et elle se subdivise en trois ensembles, diététique, pharmacopée et chirurgie'1.

À la différence de ces deux dernières disciplines, dont la vocation est essentiellement thérapeutique, la diététique sert également à prévenir les pathologies et à conserver la santé. D'emblée, donc, prévention et conservation de la santé sont définies comme faisant aussi partie des prérogatives médicales; cela signifie que le praticien peut avoir pour client aussi bien le patient que l'homme bien portant.

À partir du XIII ${ }^{\mathrm{e}}$ siècle, le savoir diététique connaît une large diffusion dans l'Occident latin, principalement à travers deux types d'ouvrages. Les conseils d'abord, ancêtres des prescriptions médicales, où, pour une affection particulière, le médecin propose au malade un régime adéquat et des préparations médicales ${ }^{2}$; la diététique n'y joue qu'un rôle thérapeutique. Les régimes de santé ensuite, terme générique qui désigne des traités voués plutôt à la prévention des maladies et à la conservation de la santé 3 . De par son contenu, cette littérature des régimes est très largement tributaire des corpus d'ouvrages grecs et arabes traduits en latin aux $\mathrm{XI}^{\mathrm{e}}$ et $\mathrm{XII}^{\mathrm{e}}$ siècles, et qui servirent de fondement à l'enseignement de l'art médical ${ }^{4}$. Toutefois, son émergence et son affirmation au sein de l'ensemble de la production médicale ne saurait s'expliquer par le seul recours aux traductions d'ouvrages anciens : il est aussi lié à la «médicalisation» progressive des sociétés médiévales et à la «professionnalisation»du métier médical ${ }^{5}$. Par ces termes, les historiens des sciences entendent l'affirmation du rôle social du médecin dans le monde urbain, percep-

${ }^{1}$ Sur ces trois parties de la médecine, voir respectivement les articles de A. Touwaide, «Stratégies thérapeutiques : les médicaments », M. McVaugh, "Stratégies thérapeutiques : la chirurgie» et P. Gil Sotres, "Les régimes de santé», Histoire de la pensée médicale, dir. M. D. Grmek, t. 1, Antiquité et Moyen Âge, trad. fr., Paris, 1995, respectivement p. 227-237, 239-255 et 257-281.

2 J. Agrimi-C. Crisciani , Les «Consilia » médicaux, Turnhout, 1993 (Typologie des sources du Moyen Âge occidental, 69).

${ }^{3}$ P. Gil Sotres, « Les régimes de santé », art. cit.

${ }^{4}$ Sur les campagnes de traductions qui permirent au savoir médical médiéval de se forger et de se diffuser via le canal universitaire, voir D. Jacquart-F. Micheau, La Médecine arabe et l'Occident médiéval, Paris, 1990 (Islam-Occident, VII).

${ }^{5}$ Sur ces questions, voir M. McVaugh, Medecine before the Plague. Practitioners and their Patients in the Crown of Aragon. 1295-1345, Cambridge, 1993 et J. Shatzmiller, Jews, Medicine and Medieval Society, Berkeley-Los Angeles-Londres, 1994. Voir également le récent numéro de Médiévales, 46, 2004, consacré à Éthique et pratiques médicales aux derniers siècles du Moyen Âge.

Cahiers de Recherches Médiévales, 13spé, 2006 
tible aussi bien à travers la rédaction de statuts qui définissent son métier et sa pratique, qui régulent ses rapports avec les patients et les questions de déontologie, que par le biais des cursus d'études et d'examens qui conditionnent l'accès à cette profession et distinguent le verus medicus du charlatan ou de l'empirique ${ }^{6}$.

La diététique a donc partie liée avec ces modifications profondes du statut et de la profession médicale aux derniers siècles du Moyen Âge. Divulgatrice de règles de vie destinées à être appliquées par le destinataire du régime, elle s'adresse aussi bien au praticien dans l'exercice de son métier qu'à un public non spécialisé. À la fois tributaire d'un héritage souvent ancien, d'un savoir théorique enseigné à l'université et de contraintes pratiques imposées par son inscription sociale, elle est l'un des lieux où se jouent les rapports, difficiles parfois, entre le médecin, figure de la contrainte, et des patients pas toujours prompts à obéir aux injonctions des praticiens voire à de simples conseils. Quels sont les fondements et les enjeux de ce savoir? Quelle fut sa diffusion et quels rapports a-t-il su nouer entre la théorie et les pratiques contemporaines? Telles sont certaines des questions qui retiendront notre attention.

\section{Les bases d'un savoir diététique}

Depuis l'Antiquité grecque, les auteurs des principaux textes médicaux ont établi des rapports étroits entre l'alimentation et la médecine: ainsi le traité hippocratique De l'Ancienne médecine (qui date de la fin du $\mathrm{V}^{\mathrm{e}}$ siècle avant J.- C.) fait même de la médecine l'héritière d'un perfectionnement de l'alimentation, et suggère que la diète des bien portants doit différer de celle des malades?

À la fin du $\mathrm{V}^{\mathrm{e}}$ siècle, un autre traité de cette collection hippocratique, intitulé $D u$ régime, définit la santé comme l'équilibre entre ce qu'on mange et ce que l'on expulse, c'est-à-dire entre ce qui sert à nourrir et ce qui n'est pas nécessaire à l'épanouissement du corps ${ }^{8}$. L'expulsion étant surtout facilitée par l'exercice physique, le texte règle les rapports entre aliments et mouvement. Mais l'auteur insiste aussi sur d'autres facteurs liés à l'environnement (comme les saisons ou la qualité de l'air par exemple) qui, selon lui, jouent un rôle dans le maintien de l'homme en bonne santé.

De ces rapports entre la santé et la maladie d'un côté, l'alimentation et plus largement l'environnement naturel de l'autre, découle le double sens du mot diaeta utilisé au Moyen Âge et d'où provient le terme «diététique » que nous employons : dans une acception étroite, la diète prend le sens de régime alimentaire; elle est alors constituée des seuls préceptes relatifs à l'alimentation et à la boisson; dans un sens large, diététique est synonyme de "régime de vie » et regroupe un ensemble de facteurs déterminants pour la conservation de la santé ou pour son recouvrement.

\footnotetext{
${ }^{6}$ Outre les travaux cités à la note précédente, voir D. Jacquart, La Médecine médiévale dans le cadre parisien, XIV $-X V^{e}$ siècle, Paris, 1998 (Penser la médecine); K. Park, Doctors and Medicine in early Renaissance Florence, Princeton, 1985.

${ }^{7}$ Hippocrate, De l'ancienne médecine, éd. et trad. de J. Jouanna, Paris, 1990 (Collection des Universités de France).

${ }^{8}$ Hippocrate, Du régime, éd. de R. Joly, Berlin, 1984 (Corpus medicorum Graecorum).
} 
Ces paramètres essentiels sont appelés dans le langage des médecins médiévaux « choses non naturelles » pour les distinguer des «choses naturelles » (ou composantes physiologiques du corps) et des «choses contre nature» ou maladies. $\mathrm{Au}$ nombre de six, ces facteurs sont de nature psychologique, physiologique ou encore liés à l'environnement naturel : l'air, l'alimentation et la boisson, le mouvement et le repos, le sommeil et la veille, ce qui est avalé et ce qui est expulsé et les passions de l'âme, telles sont les six catégories de "choses non naturelles", souvent accompagnées par des considérations sur les bains, l'activité sexuelle ou encore la saignée préventive?.

Les conceptions aussi bien conservatoires que thérapeutiques du rôle que jouent les «choses non naturelles »s'expliquent par les définitions antiques et médiévales des notions de santé et de pathologies: le corps humain, à l'image de l'univers et des quatre éléments qui le composent, est défini par un mélange de qualités premières de chaud, de froid, de sec et d'humide. L'association deux à deux de ces qualités donne naissance à la notion de complexion, que définit Galien dans le De sanitate tuenda. Une dominante chaude et humide caractérise la complexion sanguine (à l'image de l'air), une chaude et sèche définit la complexion colérique, comme le feu, tandis que dans les complexions flegmatique (comme l'eau) et mélancolique (comme la terre), ce sont respectivement les qualités de froid et d'humide et de froid et de sec qui l'emportent.

Ce mélange de qualités premières est toutefois instable; l'alimentation, comme l'air ou tout changement climatique, ou encore le vieillissement sont autant de facteurs de son altération, puisque ces choses non naturelles sont de leur côté aussi dotées d'une complexion : la consommation d'aliments trop humides pour un homme colérique, ou l'excès d'exercices qui provoquent l'échauffement des corps pour un vieillard, sont causes de déséquilibres dans ce mélange, et donc facteurs de maladie. Surtout, en fonction de l'âge, le mélange naturel évolue et si l'enfance est considérée comme chaude est humide, la vieillesse, du fait d'une perte progressive de deux éléments fondamentaux de la croissance que sont la chaleur et l'humidité naturelle, est caractérisée par une complexion froide et sèche ${ }^{10}$.

Il est donc du devoir et des compétences du médecin de définir en premier lieu si la complexion qu'il observe chez son patient est naturelle ou altérée : en fonction du résultat, il optera pour un régime conservatoire - c'est-à-dire identique à la complexion du patient lorsque ce dernier est en bonne santé, ou pour un régime contraire à cette complexion, lorsque ce dernier est malade. Le but est ainsi de conforter la nature du patient ou de lui permettre de retrouver l'équilibre perdu.

\footnotetext{
${ }^{9}$ Cf. P. Gil Sotres, «Les régimes de santé », art. cit.

${ }^{10}$ Sur le processus du vieillissement, voir L. Demaitre, «The Care and Extension of Old Age in Medieval Medicine ", Aging and the Ages in Medieval Europe, éd. M. M. Sheehan, Toronto, 1990 , p. 3-22.
} 


\section{La littérature diététique, résultante d'une demande sociale}

Si ce savoir diététique est nécessaire au praticien dans l'exercice de son art, il suscite aussi au Moyen Âge l'intérêt d'un public étranger à la chose médicale, mais soucieux de disposer de rudiments d'hygiène. Le succès que connaît la littérature diététique reflète en effet une demande sociale puisqu'elle est souvent destinée à un public de non-spécialistes. À ce titre, elle participe d'une relation nouvelle qui s'établit entre le " professionnel » de la santé et sa clientèle et favorise la diffusion de son savoir. En effet, souvent rédigé à la demande de puissants qui réclament de leurs médecins la composition d'un tel ouvrage, le régime témoigne de l'intérêt que les patients (ou du moins une certaine élite) portent à leur santé et à la conservation de celle-ci : leur relation à la pratique médicale, de thérapeutique, se déplace ainsi vers le champ proprement conservatoire.

L'essor d'une littérature des régimes de santé offre le témoignage éclatant du succès de ce genre, au dehors du milieu proprement professionnel. Entre le XIII ${ }^{\mathrm{e}}$ et le $\mathrm{XV}^{\mathrm{e}}$ siècle, ce ne sont pas moins d'une centaine d'œuvres en latin qui sont écrites sur le sujet, pour partie rédigées par des auteurs restés anonymes, pour partie par des médecins de renom. Certains d'entre eux sont en effet connus pour de nombreuses productions intellectuelles, pour leur enseignement dans une université prestigieuse ou encore pour des fonctions exercées auprès de princes, laïcs ou ecclésiastiques.

Si l'on note une prédominance de la péninsule italienne et de l'espace français comme lieu de production d'une littérature diététique, on constate aussi qu'au gré du développement universitaire, le phénomène touche l'ensemble des grands ensembles territoriaux : l'Allemagne dès le $\mathrm{XIV}^{\mathrm{e}}$ siècle, l'Angleterre dans la seconde moitié du siècle, jusqu'à la Hongrie angevine ou la Pologne.

Le succès de cette littérature se mesure aussi à l'aune des premières traductions en langues vernaculaires dont elle fait l'objet et à celle des premières compositions effectuées directement en vulgaire. L'exemple le plus souvent cité, car l'ouvrage fut très largement diffusé au Moyen Âge, est celui du Régime du corps autrement intitulé Livre de Physique d'Aldebrandin de Sienne, un médecin d'origine siennoise installé en Champagne ${ }^{11}$. Rédigé en dialecte champenois au milieu du XIII ${ }^{\mathrm{e}}$ siècle, l'œuvre fut une première fois traduite en italien, vers 1310 par un notaire florentin, Zucchero Bencivenni ${ }^{12}$.

Au-delà d'un premier public restreint de lecteurs, commanditaires des œuvres ou destinataires obligés lorsqu'il s'agit pour l'auteur de se recommander auprès d'un homme puissant (dans le cadre de la captatio benevolentie), le texte diététique a souvent su toucher un assez vaste public. Une analyse des manuscrits conservés des œuvres rédigées entre $\mathrm{XIII}^{\mathrm{e}}$ et $\mathrm{XV}^{\mathrm{e}}$ siècles (plus de 400 pour les ouvrages en latin et

\footnotetext{
${ }^{11}$ Le Régime du corps de maître Aldebrandin de Sienne, texte français du XIII siècle, publié pour la première fois d'après les manuscrits de la Bibliothèque Nationale et de la Bibliothèque de l'Arsenal, éd. de L. Landouzy-R. Pépin, Paris, 1911.

${ }_{12}$ Éd. de R. Baldini, «Zucchero Bencivenni, La santà del corpo. Volgarizzamento del Régime du corps di Aldobrandino da Siena (a. 1310) nella copia coeva di Lapo di Neri Corsini (Laur. Pl. LXXIII 47)», Studi di lessicografia italiana, 15, 1998, p. 21-300. Sur les usages de l'italien au Moyen Âge, voir Les langues de l'Italie médiévale, dir. O. Redon, Turnhout, 2002 (L'Atelier du médiéviste, 8).
} 
parfois jusqu'à 60 exemplaires pour un seul d'entre eux), et l'édition imprimée de certains titres, dès la seconde moitié du XV $\mathrm{X}^{\mathrm{e}}$ siècle, témoignent de ce succès. L'étude enfin des inventaires de bibliothèques médiévales, mais également d'autres types de sources documentaires, tels les livres de familles par exemple, ou encore les textes culinaires ${ }^{13}$ qui divulguent à leur tour des préceptes diététiques, nous informent sur la diffusion assez ample d'une culture diététique générale, touchant prioritairement un public lettré qui a accès au livre et à sa lecture.

Souvent copiés au sein de manuscrits médicaux (puisque, en général, le livre médiéval n'est pas constitué d'un seul texte mais d'un ensemble de traités ${ }^{14}$ ), les régimes de santé constituent dans l'ensemble des opuscules plutôt brefs. Ils sont plutôt copiés dans des manuscrits de facture moyenne, peu ornés et le plus souvent sans illustrations, à l'exception notable de certains: ainsi, il est des manuscrits d'origine lombarde du Tacuinum sanitatis d'Ibn Butlân, un médecin chrétien de Bagdad du $\mathrm{XI}^{\mathrm{e}}$ siècle dont l'ouvrage fut traduit en latin sans doute au XIII ${ }^{\mathrm{e}}$ siècle, qui rivalisent d'enluminures de très belle facture ${ }^{15}$ : le texte d'origine, réduit à l'essentiel, n'évoquant que la complexion de l'aliment décrit, ses vertus et nocivités, sert surtout de prétexte à une belle illustration, en rapport plus ou moins direct avec ce qu'il est censé représenter ${ }^{16}$.

$\mathrm{Au}$ gré des nouvelles compositions, le texte diététique médiéval semble accorder toujours plus d'importance aux aspects alimentaires, l'un des facteurs qui, avec l'air, est le plus essentiel à la vie mais aussi le plus dangereux pour la santé : en effet, comme l'air via la respiration, l'aliment par ingestion pénètre directement à l'intérieur de l'organisme presque sans médiation, quand d'autres paramètres non naturels, comme l'exercice ou les bains, n'affectent que la superficie du corps. Surtout, la digestion, considérée au Moyen Âge comme un processus mécanique, transforme l'aliment en tissus, en os, ou encore en sang au terme d'un processus de cuisson dans différents organes. Cette propriété que la nourriture a de se transformer, associée à la complexification croissante de l'alimentation médiévale (avec l'essor des marchés et des échanges internationaux, l'apparition de nouveaux produits, le développement de cuisines nationales et régionales...), explique la part primordiale que les médecins confèrent, à partir du $\mathrm{XIV}^{\mathrm{e}}$ siècle, à l'alimentation au sein de leurs régimes ${ }^{17}$. Certains de ces ouvrages prennent même la forme de véritables dictionnaires alimentaires, «oubliant» d'évoquer les autres composantes non

\footnotetext{
${ }^{13}$ Pour la naissance de ce type de texte, B. Laurioux, Les Livres de cuisine médiévaux, Turnhout, 1997 (Typologie des sources du Moyen âge occidental, 77).

${ }^{14}$ Pour une étude générale, cf. Le Livre au Moyen Âge, dir. J. Glénisson, Paris, 1988.

${ }^{15}$ Édition du texte arabe : Le "Taqwìm al-Sihhha» (Tacuini sanitatis) d'Ibn Butlān : un traité médical du XI siècle, éd. de Hosam Elkhadem, Louvain, 1990.

${ }^{16}$ Voir par exemples quelques fac-similés de manuscrits enluminés du Tacuinum: L'Art de vivre en santé. Images et recettes du Moyen Âge. Le "Tacuinum sanitatis » (ms 1041) de la Bibliothèque universitaire de Liège, éd. de C. Opsomer, Liège, 1991; L'Art de vivre au Moyen Age. "Codex vindobonensis series nova 2644 » conservé à la Bibliothèque nationale d'Autriche, éd. de D. Poirion-C. Thomasset, Paris, 1995.

${ }^{17}$ Pour une vision générale, voir Histoire de l'alimentation, sous la dir. de J.-L. Flandrin et M. Montanari, Paris, 1996.
} 
naturelles ${ }^{18}$; de son côté le médecin piémontais Pantaleone da Confienza, dans les années 1470, consacre un traité diététique aux seules productions laitières, la Summa lacticiniorum, qui évoque les productions fromagères du Nord de l'Italie et de l'espace français ${ }^{19}$.

\section{Normes et comportements : du discours aux pratiques}

Prolixe dans le domaine alimentaire, le praticien médiéval, auteur de régimes de santé, est en quelque sorte obligé de tenir compte des goûts ou des habitudes alimentaires de ses contemporains $\mathrm{s}^{20}$. Mais la lecture des traités suggère que nombre de recommandations médicales s'avèrent en total désaccord avec les pratiques usuelles. Le problème se pose donc de concilier les exigences d'un savoir théorique et la nécessaire adaptation d'un discours à des réalités sociales qui entrent parfois en contradiction avec elles. Quelques exemples donnent à voir la manière dont les auteurs ont pu tenter de résoudre les difficultés.

Les fruits frais sont ainsi généralement tenus par les médecins médiévaux comme dangereux pour la santé, du fait de leur nature: leur froideur et leur excessive humidité n'autorisent guère à les ranger dans la catégorie des aliments, mais bien plutôt dans celle des médicaments ou des «aliments-médicaments » : à la différence des aliments «purs », ils ne sont en effet pas transformés au cours du processus de digestion, mais ce sont eux qui, du fait de leur complexion particulière, altèrent la complexion du consommateur, à l'image de l'action des médicaments. Aussi sont-ils surtout recommandés pour soigner certaines pathologies telles les fièvres qui se caractérisent par un excès de chaleur.

Malgré cette nature particulière, les auteurs accordent toujours une place importante aux fruits dans leurs régimes, ne serait-ce que parce qu'ils étaient consommés comme aliments par les populations médiévales. Ils apparaissent même comme ingrédients dans certaines recettes de cuisine peut-être venues du monde musulman (andalou ou oriental), telle la limonia à base de jus de citron ou la romania à partir de grenades, que l'on retrouve dans des réceptaires culinaires italiens ${ }^{21}$. Aussi, à défaut de les interdire, les médecins s'efforcent-ils de proposer des préparations qui visent, pour répondre à l'appétence de leurs clients, à en faciliter la digestion : préparations cuites ou ajouts d'édulcorant pour les fruits acides par exemple figurent parmi les conseils les plus répandus.

D'autres types de préparations témoignent aussi des rapports que le texte diététique entretient avec le propos culinaire, sans que l'on prétende définir ici qui des deux a le premier influencé l'autre. À partir du XIV ${ }^{\mathrm{e}}$ siècle surtout, au moment où

\footnotetext{
${ }^{18} \mathrm{M}$. Nicoud «L'adaptation du discours diététique aux pratiques alimentaires : l'exemple de Barnabas de Reggio ", Mélanges de l'École française de Rome-Moyen Âge, 107, 1995-1, p. 207-231.

${ }^{19}$ Formaggi del Medioevo, la "Summa lacticiniorum» di Pantaleone da Confienza, éd. d'I. Naso, Turin, 1990.

${ }^{20}$ Voir B. Laurioux, Manger au Moyen Âge. Pratiques et discours alimentaires en Europe aux $X I V^{e}$ et $X V^{e}$ siècles, Paris, 2002 (La Vie quotidienne).

${ }^{21}$ O. Redon-F. Sabban-S. Serventi, La Gastronomie au Moyen Âge. 150 recettes de France et d'Italie, Paris, 1991.
} 
apparaissent aussi les premiers ouvrages de cuisine ${ }^{22}$, on note que les préparations se font plus nombreuses dans les chapitres relatifs aux viandes et aux poissons, certains auteurs allant même parfois jusqu'à proposer de véritables recettes, à l'image de l'entreprise de Maino de Maineri. Ce maître régent de la faculté de Paris, d'origine milanaise, a introduit au sein d'une vaste somme diététique de véritables recettes de cuisine pour apprêter viandes et poissons, qui furent par la suite parfois copiées séparément du reste du traité, donnant notamment naissance à un opuscule sur les sauces $^{23}$.

D'emblée, l'auteur rappelle que les sauces ont été inventées par les gourmands pour leur volupté et non pour leur santé ; à cause d'elles, dit-il, l'homme mange plus que de raison et grâce à elles, les aliments mauvais et gâtés pour la bouche sont même rendus délicieux. Malgré ces mises en garde liminaires, Maino n'en procure pas moins à son lecteur de véritables recettes dont les vertus sont diététiques : si la dénomination des sauces d'après leur couleur (sauces vertes ou blanches par exemple) et les ingrédients utilisés situent le texte dans un registre culinaire, les différences de préparation selon les saisons (quand il faut échauffer ou au contraire rafraîchir le corps) nous ramènent du côté médical. Ainsi, les épices qui, avec le vin en hiver, permettent d'échauffer (quand le verjus en été refroidit), éléments fondamentaux dans la cuisine médiévale, sont ici avant tout mentionnées pour leur complexion naturelle : le poivre est en effet chaud au $4^{\mathrm{e}}$ degré (sur une échelle de 4 ) et le clou de girofle l'est au $3^{\mathrm{e}}$, tandis que la cannelle ne l'est qu'au $2^{\mathrm{e}}$ et le safran au $1^{\mathrm{er}}$ degré. L'introduction de ces épices dans la recette répond assurément autant à des questions de goûts qu'à une économie du mélange des qualités premières, nécessaire à la conservation de la santé.

En faisant preuve d'un intérêt évident pour la cuisine médiévale, le régime prête aussi attention aux nouvelles habitudes alimentaires, dont témoignent par exemple les chapitres consacrés aux oranges et aux citrons récemment introduits parmi les cultures et les consommations de l'Occident latin. De même, les pâtes alimentaires qui, dans les espaces italiens et plus largement méditerranéens, sont préparées et souvent exportées, font également leur entrée dans les régimes, avec parfois la variété de leurs dénominations régionales ${ }^{24}$ : ainsi un auteur émilien du $\mathrm{XIV}^{\mathrm{e}}$ siècle, Barnabas Riatinis, mentionne les vermicelles toscans, les formentini de Reggio d'Émilie ou encore les pancardelle, un type de pâtes fraîches proches des lasagnes, fabriquées à Mantoue ${ }^{25}$. Au XV $\mathrm{XV}^{\mathrm{e}}$ siècle, il sera aussi question chez d'autres auteurs italiens des lasagnes, des menudelli, peut-être proches des «menudets " provençaux, des nevole (sortes de gaufres fourrées) sans oublier les recettes de gnocchi et de raviolis : le padouan Michel Savonarole, dans un ouvrage de pratique médicale, recommande même, au milieu du $\mathrm{XV}^{\mathrm{e}}$ siècle, de préparer les pâtes avec des feuilles de persil, de sauge, de menthe, agrémentées d'un peu de fromage, voire de viande de

${ }^{22}$ B. Laurioux, Le Règne de Taillevent. Livres et pratiques culinaires à la fin du Moyen Âge, Paris, 1997.

${ }^{23}$ Éd. de L. Thorndike, «A Mediaeval Sauce-Book », Speculum, 9, 1934, p. 183-190.

${ }^{24}$ O. Redon-B. Laurioux, «La constitution d'une nouvelle catégorie culinaire ? Les pâtes dans les livres de cuisine italiens de la fin du Moyen Âge », Médiévales, 16-17, 1989, p. 51-75. F. Sabban-S. Serventi, Les Pâtes : histoire d'une culture universelle, Arles, 2001.

${ }^{25}$ M. Nicoud, «L'adaptation du discours diététique... », art. cit. 
veau s'il faut conforter la vertu naturelle, et de les cuire dans une sorte de brouet bouillant. Les vermicelles (appelées tria) sont quant à elles représentées sur les $T a$ cuina sanitatis.

Que le jugement des médecins sur ces préparations culinaires et sur ces habitudes alimentaires soit favorable ou pas, force est de constater la forte imbrication des discours culinaires et diététiques à la fin du Moyen Âge. En intégrant à ses règles des conseils de préparations et en considérant que l'apprêt culinaire est un facteur susceptible de faciliter la digestion, le médecin s'efforce de réconcilier les goûts et les règles, les pratiques de ses contemporains et le savoir dont il est le dépositaire.

En tout état de cause, à ignorer certaines de ces nouvelles habitudes, le praticien, auteur de régimes, se condamnait assurément à un discours obsolète. Mais, à les critiquer, était-il certain d'être suivi par ses patients? Ici la question de la norme que divulguent ces régimes et de son application reste ouverte et difficile à trancher : ces traités ont-ils une vocation prescriptive et les conseils diététiques conservatoires sont-ils destinés à être suivis par les lecteurs ? Les conseils d'un Francesco Sforza, seigneur de Milan au $\mathrm{XV}^{\mathrm{e}}$ siècle, à son épouse Bianca Maria convalescente, lui enjoignant de faire subir à son médecin les restrictions de la diète que ce dernier lui avait imposées, incitent à croire que le propos du médecin n'est pas toujours parole d'évangile ${ }^{26}$. Que dire alors d'un Pétrarque mettant en cause les interdictions des praticiens en matière de consommation de fruits? S'adressant à son ami, Giovanni Dondi, médecin et astrologue padouan, il lui écrit :

\begin{abstract}
À présent, j'en viens aux autres conseils que tu m'as donnés, pas tant comme les tiens, mais comme préceptes de tous les médecins. Et à cette règle [qui dit] que la pomme et tous les autres fruits sont à fuir, comme le sont l'aconit ou la ciguë. Pourquoi cette aversion, cette dépréciation pour des choses qui à l'œil sont très belles, et plus appréciées et suaves que toute autre au toucher, au goût, à l'odorat? Les mortels seraient donc tous privés de discernement et de faculté de jugement, si ce n'est ceux qui détestent les pommes et les fruits ? Et comment la nature a t-elle pu tromper les hommes en leur cachant le venin dans les choses les plus belles et les plus voyantes de sa production $?^{27}$
\end{abstract}

Ici à l'interdiction du médecin, l'humaniste oppose le primat de la nature, mais aussi celui de l'expérience, du goût et du plaisir esthétique et témoigne de la difficile application des préceptes des praticiens et des formes de désobéissance du malade. Peut-on alors raisonnablement considérer que le texte diététique engage vraiment, au-delà de la norme qu'il dicte, à une pratique diététique de tous les instants? En cas de maladie, on peut le penser lorsque le patient trop affaibli était sous la coupe de son praticien; en cas de prévention ou de conservation de la santé, c'est sans doute plus difficile à croire. Mieux vaut sans doute considérer le texte diététi-

\footnotetext{
${ }^{26}$ M. Nicoud, «Les pratiques diététiques à la cour de Francesco Sforza », Scrivere il Medioevo. Lo spazio, la santità, il cibo. Un libro dedicato ad Odile Redon, dir. B. LauriouxL. Moulinier-Brogi, Rome, 2001, p. 393-404 (I libri di Viella, 28).

${ }^{27}$ Pétrarque, Lettere senili, XII, 1, 33, 35, éd. d'A. Petrucci, Francesco Petrarca, Epistole autografe. Introduzione, trascrizione e riproduzione, Padoue, 1968, p. 45-46.
} 
que comme un texte non pas prescriptif mais normatif, au sens où il indique le moyen pour le patient d'agir en accord avec la nature et ses règles (ou mieux encore d'agir en accord avec sa nature propre), tout en lui laissant le choix de s'y conformer ou de ne pas les suivre.

Marilyn Nicoud École Française de Rome - École Normale Supérieure Lettres et Sciences Humaines 